the liposome control group RNA interference group proliferation is weak $(\mathrm{p}<0.05)$.

Conclusion 1. HIF-1a, SDF-1a and VEGF gene expression can be affected by HIF-1a siRNA in MSCs. 2. Hypoxia can made HIF-1 a, SDF-1 a and VEGF gene expression increased. 3. SDF-1 a and VEGF gene expression may be controlled by HIF-1 a in MSC. 4. Cell culture medium stimulate SMC profliction can be reduced by RNA interfence.

\section{E0099 BAICALIN PROTECTION RAT CARDIOMYOCYTES FROM ISCHAEMIA-REPERFUSION INJURY AND ANTIARRHYTHMIA VIA INHIBITING L-TYPE CALCIUM CURRENT}

doi:10.1136/hrt.2010.208967.99

Teng Wang, Jingjing Wang, Wenyun Gan, Congxin Huang. Department of Cardiology, Renmin Hospital of Wuhan University, Cardiovascular Research Institute of Wuhan University, Wuhan, China

Objective To investigate baicalin protection rat cardiomyocytes from inschemia-reperfusion injury and antiarrhythmia via blocking $\mathrm{I}_{\mathrm{Ca}-\mathrm{L}}$. Methods The degree of ischaemia-reperfusion injury was assessed by the recovery of LVDP and the magnitude of the reperfusion contracture with using approach of the Langendorff-perfused isolated rat hearts. The effects of baicalin on APs and ouabaininduced $\mathrm{DAD}$ and $\mathrm{AT}$ were performed on rat papillary muscles by conventional microelectrode technique. $\mathrm{I}_{\mathrm{Ca}-\mathrm{L}}$ was recorded via using whole-cell patch-clamp technique in enzymatically dissociated single rat ventricular myocytes.

Results Compared with the pre-ischaemic control, baicalin could concentration-dependently improved recovery of LVDP, and reduced the lever of reperfusion contracture, and occurrence of arrhythmias. Baicalin significantly shortened $\mathrm{ADP}_{20}, \mathrm{ADP}_{50}$ and $\mathrm{APD}_{90}$ in rat papillary muscles. Ouabain could apparently induced the DAD and TA in rat papillary muscles. With administration of baicalin, the electrophysiological parameters of ouabain-induced $\mathrm{DAD}$ and TA were markedly inclined to difficult occurrence. It illustrated that baicalin might inhibit influx of $\mathrm{I}_{\mathrm{Ca}-\mathrm{L}}$. Baicalin significantly inhibited $\mathrm{I}_{\mathrm{Ca}-\mathrm{L}}$ in a voltage-dependent and concentration-dependent procedure, with an $\mathrm{IC}_{50}$ value of $27.7 \pm 1.9 \mu \mathrm{mol} / \mathrm{l}\left(\mathrm{E}_{\max }\right.$ and $\mathrm{nH}$ were $115.2 \pm 3.3 \%$ and $1.07 \pm 0.05$, respectivly). Moreover, baicalin shifted the I-V curve of $\mathrm{I}_{\mathrm{Ca}-\mathrm{L}}$ upwards. According to statistic kinetic data, it was suggested that baicalin especially inhibit the $\mathrm{I}_{\mathrm{Ca}-\mathrm{L}}$ by eliciting a negative shift of the steady-state inactivation without affecting the slop factor. To the effect of baicalin on the speed of $\mathrm{I}_{\mathrm{Ca}-\mathrm{L}}$ recovery from inactivation, our data indicated that the time courses of recovery were prolonged markedly $(p<0.01$ compare with control group, respectively).

Conclusions Baicalin improved cardioprotection effects on ischaemia-reperfusion injury and decreased the occurrence of ouabain-induced DAD and TA, thus inhibited $\mathrm{I}_{\mathrm{Ca}-\mathrm{L}}$. The effects of baicalin on inhibiting $\mathrm{I}_{\mathrm{Ca}-\mathrm{L}}$ might contribute to baicalin antagonising ischaemia-reperfusion injury and arrhythmia.

\section{Q0100 ASSOCIATION BETWEEN CREG GENE POLYMORPHISMS AND CORONARY ARTERY DISEASE IN THE HAN POPULATION OF NORTH CHINA}

doi:10.1136/hrt.2010.208967.100

Tao Wang, Xiaolin Zhang, Yaling Han, Chenhui Yan, Mingfang Huang. Shenyang Northern Hospital

Introduction The purpose of the present study was to assess the possible association between CREG and CAD in the Han population of North China.

Methods ALL five selected SNPs were genotyped in 1161 patients with angiographically documented CAD and 960 control subjects free from $\mathrm{CAD}$ who had normal coronary angiograms. Patients and controls were unrelated individuals of Han Chinese from the northeast region of China, genotype analyses were performed additive, dominant and recessive models. Binary logistic regression was used to control for the presence of vascular risk factors both in genotype and haplotype analyses.

Results Genotype frequencies of the five examined polymorphisms were similarly distributed between CAD group and controls $(p>0.05)$. Further haplotype analysis also found no significant differences in the distributions between CAD group and controls ( $p>0.05)$.

Conclusion This study did not show a statistically significant association between common variants of CREG and CAD in northern Chinese Han population.

\section{e0101 INTERLEUKIN-17A GENE VARIANTS AND RISK OF CORONARY ARTERY DISEASE:A LARGE ANGIOGRAPHY- BASED STUDY}

doi:10.1136/hrt.2010.208967.10

Xiaolin Zhang, Fang Pei, Yaling Han, Chenhui Yan, Mingfang Huang, Tao Wang. Shenyang Northern Hospital

Objective Recent studies have also revealed that interleukin (IL)-17A plays a key role in atherosclerosis and its complication, but the relationship of its common variants with coronary artery disease (CAD) has not been extensively studied.

Methods We systematically screened sequence variations in the IL17A gene and designed an angiography-based case-controlled study consisting of $1031 \mathrm{CAD}$ patients and 935 control subjects to investigate the association between the selected polymorphisms of IL-17A gene and CAD risk in Chinese Han population.

Results Frequencies of IL17A rs8193037 GG homozygote and G allele were significantly higher in the patient group than those in the control group $(p<0.001 ; O R=0.68 ; 95 \%$ CI 0.54 to 0.85$)$. Stratification analysis showed that the IL17A rs8193037 G allele significantly increased the risk of $\mathrm{CAD}$ only among male subjects ( $p=0.001 ; O R=0.63 ; 95 \%$ CI 0.47 to 0.83 ). After adjustment for conventional risk factors, binary logistic regression analysis showed that the the $G$ allele carriers (GG $+A G$ ) had significantly increased $\mathrm{CAD}$ risk compared with the AA homozygotes (adjusted $\mathrm{p}<0.001$; OR 0.43 ; $95 \%$ CI 0.33 to 0.58 ). ELISA showed augmented IL17A production in serum of the AMI patients.

Conclusions Based on our data, we speculated that rs8193037 of IL17A is associated with CAD risk in Chinese Han population and G allele of rs8193037 may be an independent predictive factor for $\mathrm{CAD}$.

\section{e0102 EXPRESSION OR SECRETION OF IL-34 AND IL-35 IN THE PERIPHERAL BLOOD MONONUCLEAR CELLS FROM PATIENTS WITH DILATED CARDIOMYOPATHY}

doi:10.1136/hrt.2010.208967.102

Huang Sisi, Wu Weifeng, Lin Song, Huang Yanlan. The First Affiliated Hospital of Guangxi Medical University

Objective The aim of this study was to observe the level of interleukin (IL)-34 and IL-35 in peripheral blood mononuclear cells (PBMCs) with dilated cardiomyopathy (DCM), and explore the role of IL-34 and IL-35 in human DCM.

Methods 30 patients with DCM and 30 normal adults as control were studied. IL-34 and the subunit Epstein-Barr virus-induced gene 3(EBI3) of IL-35 mRNA expression in PBMCs were detected by reverse transcription-PCR (RT-PCR). IL-34 and IL-35 protein level in plasma were measured with ELISA.

Result (1) Results showed that the IL-34 mRNA level or its protein level was significantly elevated in DCM patients compared with 